ENCYCLOPEDEE Encyclopédie berbère

BERBERE

$27 \mid 2005$

27 | Kairouan - Kifan Bel-Ghomari

\title{
Kifan Bel-Ghomari
}

C. Roubet et S. Hachi

\section{OpenEdition}

Journals

Édition électronique

URL : http://journals.openedition.org/encyclopedieberbere/1364

DOI : 10.4000/encyclopedieberbere.1364

ISSN : 2262-7197

\section{Éditeur}

Peeters Publishers

Édition imprimée

Date de publication : 1 août 2005

Pagination : 4245-4250

ISBN : 2-7449-0538-0

ISSN : 1015-7344

Référence électronique

C. Roubet et S. Hachi, « Kifan Bel-Ghomari », Encyclopédie berbère [En ligne], 27 | 2005, document K60, mis en ligne le 01 juin 2011, consulté le 24 septembre 2020. URL : http://journals.openedition.org/ encyclopedieberbere/1364; DOI : https://doi.org/10.4000/encyclopedieberbere.1364

Ce document a été généré automatiquement le 24 septembre 2020

(c) Tous droits réservés 


\title{
Kifan Bel-Ghomari
}

\author{
C. Roubet et S. Hachi
}

1 Au Maroc oriental, dans les contreforts du Moyen Atlas, non loin de la ville de Taza (585 $\mathrm{m}$ d'altitude) s'ouvre un important réseau de grottes (à $565 \mathrm{~m}$ d'alt.). L'une d'elles porte le nom du lieu-dit: Kifan bel-Ghomari; elle se situe près d'une ancienne porte de la ville nommée Bab Djemâ-Foukounia et à proximité des vestiges d'un mur d'enceinte désigné comme «Bastioun ». L'ancien sentier muletier sert de chemin principal pour y accéder. C'était à peu près le signalement que faisait en 1917 le lieutenant J. Campardou qui avait découvert et exploré les lieux en 1915 et 1916 et qui exposait ses résultats dans une étude, tombée à tort dans l'oubli.

On doit surtout à trois savants naturalistes F. Doumergue (1917), P. Pallary (1918-1919) et à G. Lecointre (qui participa aux recherches, in Balout 1955: 369, note 3) d'avoir avalisé et soutenu les travaux du Lt. J. Campardou $(1917,1921)$ en examinant les lieux fouillés et les documents recueillis que citèrent bien plus tard R. Vaufrey (1955), L. Balout (1955) et G. Camps (1974). Aucune autre recherche n'a été entreprise dans la grotte et aux alentours. Pourtant de nombreux sujets d'intérêt ont été très tôt signalés (Campardou 1917, 1921; Basset 1925). Ces études permettent de comprendre l'attachement des populations berbères à cette région boisée, pourvue en sources, ayant eu un habitat troglodytique dans les travertins et conservant une nécropole dans les cavités naturelles. L'intérêt de cette grotte est d'avoir maintenu en superposition non contestable et dans un bon état de conservation deux niveaux archéologiques distincts : l'un moustérien, l'autre ibéromaurusien. Sans hésitation, le Lt. J. Campardou les isola et établit plusieurs croquis précisant l'emplacement de ses découvertes. Elles étaient exceptionnelles et les travaux minutieux.

3 La collection, déposée au Musée Demaeght d'Oran, permit à F. Doumergue $(1917,1936)$ d'identifier et de lister le matériel faunique des deux ensembles culturels, s'appuyant notamment sur ses collections d'Oranie, puis à P. Pallary d'examiner les séries lithiques marocaines (1918-1919). Pour l'une des deux industries, il devait conclure qu'il approuvait le diagnostic d'Ibéromaurusien fait par J. Campardou, fondé sur un examen comparatif avec l'industrie de l'Abri de la Mouillah (Barbin 1910, 1912). Acceptés 
ensuite par tous les autres auteurs, ces résultats ne furent jamais contredits, ni développés.

\section{État de la Grotte (Campardou 1917)}

La grotte creusée dans des tufs calcaires friables, à Pulmonés actuels, s'ouvre à l'est. Sur la paroi gauche de l'entrée ( $4 \mathrm{~m}$ x 3,50 m), mais à l'extérieur et en hauteur, on remarque une sculpture circulaire d'1,3 m environ, en relief, suggestive d'une « représentation solaire ». On entre dans une vaste salle de $10 \mathrm{~m}$ de diamètre sur $5 \mathrm{~m}$ de hauteur, occupée par un pilier rocheux central de $2 / 3 \mathrm{~m}$ de diamètre; d'autres ramifications ou galeries s'enfonçaient et s'étendaient dans ces formations, certaines parois paraissant modifiées. L'observation de toutes les parois a conduit le fouilleur à reconnaître des cavités naturelles et d'autres à parois taillées. J. Campardou nous rappelle que l'exploitation des tufs du massif au cours du temps a été manifeste et prolongée, si bien que la grande salle de la grotte a été partiellement vidée. Toutefois, l'obstruction des galeries due à d'importants dépôts anthropiques, contenant des silex et des faunes, vint apporter des arguments en faveur du maintien in situ des accumulations préhistoriques, non remaniées.

\section{Travaux préliminaires 1915 - Campagne de fouilles 1916}

$5 \quad$ Les travaux préliminaires ont consisté à faire des sondages vers le nord de la salle et autour du pilier, sans grand succès. Les fouilles conduites en tranchées (N.-S.) ont dégagé les sédiments résiduels de l'entrée, puis atteint vers le sud ceux des galeries I et II.

6 Quatre couches ont été individualisées, qui servirent de repères aux travaux ultérieurs : - A : 0, $40 \mathrm{~m}$, couche superficielle remaniée avec tessons de poterie,

- B : 0, $60 \mathrm{~m}$, couche moyenne contenant des sépultures, des objets en fer, des fragments de poterie, quelques silex et ossements,

- C : $0,40 \mathrm{~m}$, couche profonde grise à jaunâtre, contenant des pierres de foyers, des cendres indurées, des ossements, des silex, des outils en os poli, des coquillages,

- $\mathrm{D}: 0,90 \mathrm{~m}$, sables gréseux rougeâtre à ossements.

7 La couche $\mathrm{C}$ n'a pas été affectée par les ensevelissements postérieurs. "Sa stratification est d'une régularité parfaite... Elle est constituée par un dépôt brécheux, de couleur gris clair, dans lequel les ossements d'animaux et les cendres de foyers sont souvent agglomérés avec des silex taillés et des coquilles d'hélix. Aucune poterie n'y a été rencontrée. Ce dépôt $C$, absent de la cavité principale, se ramifie dans les galeries I, II, et III » (Campardou, 1917 : 11).

8 La campagne de fouilles de 1916 s'est déplacée vers les galeries III et IV, la Salle des Stalactites et le Boyau des Orgues. Sans entrer dans le détail topographique de ces lieux aux parois couvertes de calcite, de stalactites et de stalagmites, assez difficiles à suivre, rappelons que le schéma stratigraphique ci-dessus a été retrouvé. La galerie IV (6 m x 2 $\mathrm{m} \times 4 / 5 \mathrm{~m}$ ) devait communiquer avec la salle principale, la Salle des Stalactites et le Boyau d'Orgues, avant d'être comblée par les sédiments. 


\section{Stratigraphie}

\section{Le Moustérien}

9 La couche argileuse D de couleur rouge (p. 18) a été individualisée et fouillée en détail à droite de l'entrée (Pl. II), et dans la galerie IV en particulier. Dans cette galerie une succession particulière des derniers dépôts argileux de la couche $\mathrm{D}$ a été observée. Ceux-ci se seraient déposés en alternance avec les premiers dépôts de la couche postérieure C. J. Campardou à bien noté cette intercalation, rattachant sur des bases colorimétriques et contextuelles, les minces dépôts C1 (cendreux), D1 (argileux) et C2 (cendreux), D2 (argileux) respectivement aux couches C et D (Pl. II).

"On a pu recueillir une très importante collection d'ossements d'animaux propres à la couche $D$, mais aussi un nouveau lot de silex dont l'intérêt... est peut être supérieur à celui de la couche $C$. On a pu établir que la couche D occupait toute la partie inférieure de la grotte et de ses ramifications» (p. 13). « Comme l'industrie lithique et les ossements semblaient présenter, dans les parties les plus profondes du dépôt de remplissage, un faciès plus archaïque, on a été amené à désigner par la lettre $E$, les parties inférieures de la couche $D$. Un foyer y a été, du reste, reconnu. $A u$ contraire aucune trace d'habitat, même temporaire, n'a été rencontré dans la couche $D$, malgré la présence de silex, d'ailleurs sporadiques, au milieu des ossements d'animaux» (p. 15-16). La couche D a aussi été retrouvée et fouillée dans la Salle des Stalactites. "Dans ces diverses cavités, les dépôts n'avaient pas partout la même importance. Tandis que la galerie IV était presque entièrement comblée, le colmatage de la Salle des Stalactites était, au contraire, peu important. Quant au Boyau des Orgues, dont l'entrée se trouvait presque obstruée... il ne contenait aucun dépôt $»($ p. 15).

11 L'épaisseur totale des couches $\mathrm{C}$ ( $\mathrm{C} 1: 0,90 \mathrm{~m}$ et $\mathrm{C} 2: 0,50 \mathrm{~m}$ ), D (D1:0,65 m et D2:2,25 m) et $\mathrm{E}(1,10 \mathrm{~m})$, atteignait 5,40 $\mathrm{m}$ (Campardou, p. 16$)$.

12 Le foyer 6 découvert en $\mathrm{E}$ était conservé sous une épaisseur totale de 4,25 $\mathrm{m}$ de sédiments, mais « sous deux mètres environ de dépôts appartenant à la couche D. Il était formé d'une simple lentille de cendres de 0,50 $\mathrm{m}$ de diamètre environ et de 0,10 $\mathrm{m}$ d'épaisseur, il était entouré de quelques ossements mêlés à des coquilles d'hélix. Les silex grossiers trouvés au voisinage du foyer étaient fortement cacholonnés et paraissaient avoir subi l'action du feu » (p. 19).

13 Sur les Pl. VII et VIII figurent des racloirs sur lames d'excellente facture moustérienne.

14 La faune identifiée par F. Doumergue (1917) (équivalences établies par R. Vaufrey 1955, p. 279) comprend : le renard (Vulpes atlantica); la hyène tachetée (Hyaena striata); le lion (Felix leo); la panthère (Felix pardus); le chat ganté (Felix libyca); l'ours des cavernes (Ursus spaeleus); le hérisson d'Algérie (Erinaceus algirus); le lièvre (Lepus kabylicus); le zèbre (Equus mauritanicus); l'âne (asinus africanus); le rhinocéros subinerme (rhinocéros simus); le rhinocéros de Merck (rhinoceros Mercki); le sanglier (Sus scrofa); le petit bœuf d'Ibérie (bos ibericus); le grand bœuf (Bos primigenius = syncerus); le mouflon (Ammotragus lervia); l'oryx (Oryx leucoryx); la gazelle dorcade (Gazella dorcas); la gazelle atiantique (Gazella atlantica); gazelle de Cuvier (Gazella cuvieri); l'ouach (Bubalis boselaphus); le cerf élaphe (Cervus elaphus). Pour les Carnivores, consulter aussi l'étude de A. Mas (1952 et 1955). 


\section{L'Ibéromaurusien} Rappelons que les croquis des six planches furent esquissés sur place, sous la lampe,
dans l'inconfort et le souci d'exactitude. Le rocher nu a été atteint partout (p. 16). Toutefois, on ne sait s'il subsiste des dépôts masqués, susceptibles d'être encore fouillés.

Cet ensemble culturel a été notamment observé dans le foyer 4 , « derrière un rideau stalagmitique masquant l'entrée de la galerie III,... la coupe montre en outre le plancher stalagmitique séparant en ce point la couche D des niveaux supérieurs » (p. 19).

La couche cendreuse et grise $C$ a livré cinq foyers, situés près des parois, non loin de l'entrée (p. 19). Auprès d'eux, de grandes quantités d'ossements et de coquilles d'Hélix et d'Unios se sont accumulées. De nombreux silex taillés s'y trouvaient mêlés.

Ce sont pour la plupart des nucleus, des produits de débitage, des lames et lamelles à bord abattu ayant une extrémité aiguë, des lamelles à troncatures simples ou doubles, des pièces à coches etc. Les dessins des Pl. VII et VII sont à eux seuls démonstratifs, mais leur représentation quantitative est insuffisante. Il s'agit de vingt pièces typologiquement conformes à ce que divers auteurs ont dessiné et décrit, bien plus tard, comme Ibéromaurusien. Ces lamelles «paraissent tout à fait assimilables à celles observées... à la station de la Mouillah» (p. 20-21). Pourtant, aucune étude n'est venue détailler la composition de cet équipement lithique, aucun décompte n'a même été établi ; L. Balout estime à un millier le nombre de pièces typiques et à environ 4000 , l'ensemble des déchets (Balout 1955, p. 369). P. Pallary n'a pas jugé bon de publier les informations tirées de ses observations comparatives (1918-1919, p. 94-96).

Signalons encore la présence de l'os poli, de l'oxyde rouge de fer et d'un galet plat. Le test d'œuf d'autruche est totalement absent (Vaufrey, 1955 : 279).

Les documents fauniques de la couche C déterminés par E Doumergue (1917) sont moins nombreux que ceux de la couche $D$, ce sont ceux du renard, de la hyène rayée, du lion, du lièvre, du cheval ou zèbre ( ?), du sanglier, du petit et du grand bovidé, du mouton ( ?), de la chèvre (?), de l'oryx, de la gazelle dorcade, de la gazelle de cuvier, de l'antilope bubale et du chameau.

Quelques rares ossements humains proviennent aussi de la couche C (p. 18). Ils n'ont pas été décrits.

\section{Conclusion}

Rien n'est à écarter, aujourd'hui encore, dans la publication pionnière du Lt. J. Campardou. Tout y est exprimé et dessiné avec détail et précision. Cette étude n'est pas seulement à relire, elle est désormais à reprendre.

Rappelons que les croquis des six planches furent esquissés sur place, sous la lampe,

La genèse de l'édification sédimentaire de cette grotte a été reconstituée par le Lt. J. Campardou (p. 24) : "La grotte de Kifan bel-Ghomari semble se comporter comme une cavité mixte dans laquelle des couches d'habitat seraient venues se superposer à des couches de remplissage.

"La couche $D$ et ses parties profondes (couche E) sont en effet, des couches de dépôt limoneux qui ont été formées par les eaux et qui sont venues tout d'abord remplir le fond des galeries, là où des obstacles,... permettaient au colmatage de s'effectuer. [...] Pendant la constitution de ces dépôts de remplissage, la caverne a été habitée surtout par les gros 
carnassiers qui y ont laissé les restes de leurs repas. La présence d'un grand nombre de pièces de l'hyène et des débris de plusieurs ours ne laissent aucun doute à cet égard. Le foyer de la couche $E$ doit être considéré comme un événement temporaire... Situé dans un endroit relativement obscur, il semble marquer une occupation passagère de la cavité au commencement de son remplissage " «La couche D semble représenter l'industrie de l'époque moustérienne» (p. 25).

«au-dessus... après une interruption d'habitat par l'homme... est venue se superposer la couche $C$, qui constitue un niveau d'habitat parfaitement déterminé... Cette couche paraît synchronique de celle de la grotte de la Mouillah... d'âge Ibéromaurusien » (p. 25-26). Elle est «caractérisée par l'importante collection de petites lames à dos retouché et les foyers supérieurs 1 à 5 ».

Retenons aussi quelques mots clé : travertins, sources, forêts.

Couches E et D : dépôt limoneux, plancher stalagmitique, carnivores et bref impact anthropique Moustérien : halte.

Couche $C$ : foyers et dépôts d'un habitat de chasseurs Ibéromaurusiens.

La région de Taza est connue pour son attrait et ses ressources dès le Paléolithique.

Nos connaissances locales du Pléistocène supérieur (40 derniers millénaires) sont à établir, elles se réduisent pour l'instant à quelques indices (couches E et D de Kifan belGhomari).

Les chasseurs Ibéromaurusiens de Kifan bel-Ghomari ont séjourné dans un milieu un peu plus froid que l'actuel, où croissaient peut être le pin Laricio et le cèdre. Mais contrairement à leurs cousins des Babors de l'Algérie, chassant surtout le mouflon (Hachi, 1999), ceux-là semblent avoir eu des comportements plus opportunistes et moins sélectifs.

Dans ce secteur du Maroc oriental situé à moins de $100 \mathrm{~km}$ de la mer, aucune datation n'est disponible. Seul repère d'importance régionale : la grotte ossuaire de Taforalt, au nord-ouest de Taza. Elle a livré neuf niveaux d'Ibéromaurusien : $8850 \mathrm{BC}$ pour le niveau II et 13750 BC pour le niveau XII (Camps 1974) qui attestent de la longévité de cette culture et du pouvoir d'attraction de ce site, pour des populations de Cro-Magnon: Hommes et Femmes de Mechta-Afalou, essaimées le long de la Moulouya, voire jusqu'à Taza. Taforalt fut sans nul doute comme Afalou Bou Rhummel, un haut lieu de mémoire culturelle (Hachi 1999 ; Hachi et Roubet 2003).

Il y a quelques décennies déjà, on s'accordait à reconnaître que l'Oranie méditerranéenne avait été un foyer de culture ibéromaurusienne, mais son originalité et son importance restent encore à établir. En somme, rien ne nous autorise à privilégier aujourd'hui l'hypothèse d'une éventuelle pénétration d'est en ouest des Hommes de Mechta-Afalou, en utilisant la trouée de Taza.

Attendons les résultats d'autres travaux régionaux. Ils nous réservent d'autres réponses.

\section{BIBLIOGRAPHIE}

ARAmbourg C, 1938. Mammifères fossiles du Maroc. Mém. de la Soc. des Sc. Nat. du Maroc, nº XLVI. 
BALOUT L., 1955. Préhistoire de l'Afrique du Nord. Essai de chronologie. Paris, AMG.

BASSET H., 1925. « Les Troglodytes de Taza ». Hespéris, t. V, pp. 427-442.

BARBIN A., 1910. « Fouilles des abris préhistoriques de la Mouillah, près Marnia ». Bull. de la Soc. de Géogr. et d'Archéol. de la Province d'Oran, t. XXX, pp. 77-90.

BARBIN A., 1912. « Fouilles des abris préhistoriques de la Mouillah, près Marnia, deuxième campagne ». Bull, de la Soc. de Géogr. et d'Archéol. de la Province d'Oran, t. XXXII, pp. 389-402.

CAMPARDOU LT. J., 1917. « La nécropole de Taza (Maroc) ». Bull, de la Soc. de Géogr. et d'Archéol. de la Province d'Oran, t. XXXVII, pp. 291-329.

CAMPARDOU LT. J., 1921. « Notes archéologiques sur la région de Taza (Maroc) ». Bull, de la Soc. de Géogr. et d'Archéol. de la Province d'Oran, t. XLI, pp. 173-194.

CAMPARDOU LT. J., 1917. « La grotte de Kifan bel-Ghomari à Taza (Maroc) ». Bull, de la Soc. de Géogr. et d'Archéol. de la Province d'Oran, t. XXXVII, pp. 5-26.

CAMPS G., 1974. Les civilisations préhistoriques de l'Afrique du Nord et du Sahara. Doin.

DOUMERGUE F., 1936. «Inventaire de la section de Préhistoire du Musée Demaeght, à Oran ». Bull, de la Soc. de Géogr. et d'Archéol. de la Province d'Oran, t. LVII, 53-54, nº 255.

DOUMERGUE F., 1917. « Faune » in Campardou Lt. J. « La grotte de Kifan bel-Ghomari à Taza (Maroc) ». Bull, de la Soc. de Géogr. et d'Archéol. de la Province d'Oran, t. XXXVII, pp. 5-26 (tableau p. 17).

ENNOUCHI E., 1953. « Complément de faune de Kifan bel-Ghomari (Taza)». Comptes rendu mens, de la Soc. des Sc. Nat. du Maroc, t. XIX, pp. 26-27.

HACHI S. 1999. L'Homme de Mechta-Afalou, Cro-Magnon de l'Afrique du Nord durant les 20 derniers millénaires : évolution culturelle et devenir. Thèse d'habilitation à diriger des recherches, Université P. Valéry III, Montpellier, 4 volumes.

HACHI S. et ROUBET C, 2003. « Les premières inhumations en nécropoles de l'Homme de MechtaAfalou ». Dossiers d'Archéologie, $n^{\circ}$ 282, pp. 16-21.

MAS A., 1952. Contribution à l'étude des Carnivores fossiles de Kifan bel-Ghomari (Taza). Diplôme d'études supérieures de géologie, Rabat.

MAS A., 1955. Contribution à l'étude des Carnivores fossiles de Kifan bel-Ghomari (Taza). Rabat, Mém. soc. sci. nat. et phy. du Maroc, 2 (1), 47 p.

PALLARY P., 1918-1919. « Revue de Préhistoire Maghrébine (1914-1917) ». L’Antrop., t. XXIX, pp. 89-104 (94-96).

VAUFREY R., 1955. Préhistoire de l'Afrique. Tome 1, Le Maghreb. Paris, Masson.

\section{INDEX}

Mots-clés : Ibéromaurusien, Maroc, Moustérien 